\title{
Applicability of the Sparse Temporal Acquisition Technique in Resting-State Brain Network Analysis
}

\author{
(D) N. Yakunina, T.S. Kim, W.S. Tae, S.S. Kim, and DE.C. Nam
}

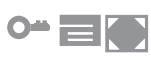

\begin{abstract}
BACKGROUND AND PURPOSE: The ability of sparse temporal acquisition to minimize the effect of scanner background noise is of utmost importance in auditory fMRI; however, it has considerably lower temporal efficiency and resolution than the conventional continuous acquisition method. The purpose of this study was to determine whether sparse sampling could be applied to resting-state research by comparing its results with those obtained by using continuous acquisition.
\end{abstract}

MATERIALS AND METHODS: We identified resting-state networks by using independent component analysis and measured their functional connectivity strength in 14 healthy subjects who underwent two 6-minute sparse (60 volumes) and continuous ( 360 volumes) imaging sessions. To account for the sample size difference, an additional continuous dataset was generated by temporally matching the continuous dataset to 60 volumes of the sparse dataset.

RESULTS: Consistent resting-state network maps were produced through all 3 datasets. Scanner background noise did not appear to affect the spatial constitution of the networks, whereas a larger sample size influenced it substantially. The strength of the intranetwork connectivity was similar through the 3 datasets.

CONCLUSIONS: Our results indicated that continuous acquisition is a recommended technique that should be applied in most of the resting-state studies due to its superior temporal efficiency and increased statistical power. The use of sparse temporal acquisition should be restricted to very particular conditions when continuous scanner noise is unacceptable.

ABBREVIATIONS: $C A=$ continuous acquisition; DMN = default mode network; $\mathrm{RSN}=$ resting-state network; SBN = scanner background noise; STA = sparse temporal acquisition; ICA = independent component analysis

$\mathbf{R}$ esting-state fMRI may be a better alternative for studying mechanisms that underlie certain auditory disorders than stimulation-based studies. For example, in the case of tinnitus, it may be preferred to investigate spontaneous brain activity in the absence of any external auditory stimulation when tinnitus is

Received November 1, 2014; accepted after revision August 17, 2015.

From the Institute of Medical Science (N.Y.), Department of Radiology (S.S.K.), and Department of Otolaryngology (E.C.N.), Kangwon National University, School of Medicine, Chuncheon, Republic of Korea; and Neuroscience Research Institute (N.Y., W.S.T., S.S.K., E.C.N.) and Department of Otolaryngology (T.S.K.), Kangwon National University Hospital, Chuncheon, Republic of Korea.

This research was supported by the Basic Science Research Program through the National Research Foundation of Korea, funded by the Ministry of Education (2014RIA1A4A01003909).

Please address correspondence to Eui-Cheol Nam, MD, Department of Otolaryngology, School of Medicine, Kangwon National University, One Kangwondaehakgil, Chuncheon, Kangwon-do, 200-701, Republic of Korea; e-mail: birdynec@ kangwon.ac.kr

- Indicates open access to non-subscribers at www.ajnr.org

$\equiv$ Indicates article with supplemental on-line tables.

Indicates article with supplemental on-line photos.

http://dx.doi.org/10.3174/ajnr.A4554 more prominent because no acoustic masking or distraction is present. A few recent studies examined resting-state functional connectivity in patients with tinnitus; however, all of the studies used the conventional continuous acquisition (CA) method, which constantly produces scanner background noise (SBN) and may mask tinnitus acoustically or may even cause residual inhibition for several minutes. ${ }^{1-4}$

Sparse temporal acquisition (STA), in contrast, reduces the effect of SBN by using long interacquisition intervals, which allows hemodynamic response induced by acquisition noise to decay completely or close to baseline at the time of the next image acquisition. ${ }^{5}$ Practical use of STA in resting-state research has barely been explored. The trade-off required to minimize the SBN effect by using the STA approach is a lower temporal efficiency that allows substantially fewer volumes to be obtained within a limited imaging time, which could affect the analysis of functional maps and functional connectivity. Questions such as whether STA can provide resting-state results comparable with those widely and consistently obtained to date by using CA techniques and, if the results are different, whether the discrepancy is due to 


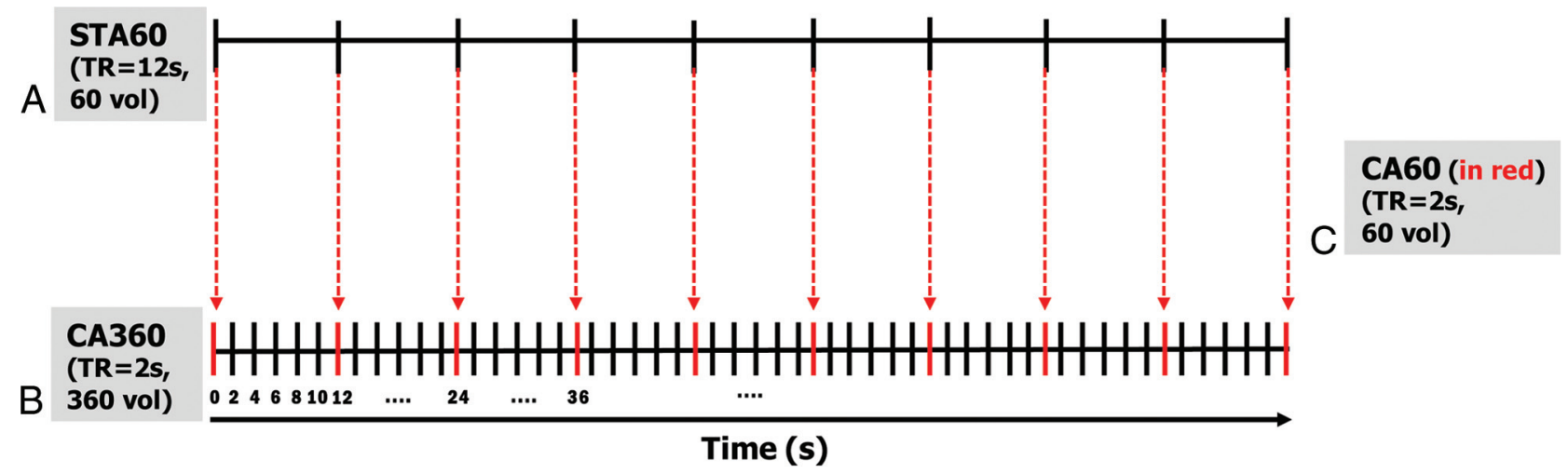

FIG 1. Construction of sparse and continuous image acquisition datasets. A, STA (STA60) design. Sixty images were acquired every 12 seconds. $B, C A(C A 360)$ design. Three-hundred and sixty images were acquired every 2 seconds. C, CA60 design. The number of volumes in the CA360 data set was matched with the STA60 dataset by selecting every sixth image that corresponded in time to the STA60 acquisitions (CA60; marked with red arrows).

the SBN effect or differences in sample size between the 2 acquisition methods, remain unanswered.

In this study, we separated the effects of SBN and sample-size differences when comparing the STA and CA techniques. Specifically, we compared resting-state networks (RSNs) obtained by the 2 acquisition methods in terms of spatial map constitution and intranetwork functional connectivity strength to establish the applicability of STA to fMRI studies that explore auditory pathophysiology related to alterations in the brain resting state, such as tinnitus or other forms of auditory hyperactivity, in which the noisy environment of CA is unacceptable.

\section{MATERIALS AND METHODS Subjects}

This study was approved by the institutional review board of Kangwon National University Hospital. All the subjects gave written informed consent before participation in this study. Fourteen healthy subjects (mean age, $30.6 \pm 4.7$ years; all right-handed; 8 men) with normal hearing ( $<20 \mathrm{~dB}$ hearing level in a standard audiometric frequency range of $250-8000 \mathrm{~Hz}$ ) participated in the study. The subjects had no known auditory, neurologic, or neuropsychologic disorders. Before the fMRI session, all the subjects underwent pure-tone audiometry and were examined for loudness discomfort level and dynamic range.

\section{Data Acquisition}

Imaging was performed by using a $3 \mathrm{~T}$ MR imaging scanner (Achieva TX; Philips Healthcare, Best, the Netherlands) with a 32-channel SENSE head coil. Coronal 3D T1-weighted high-resolution structural images of the whole brain were acquired for anatomic orientation (TR, 9.8 milliseconds; TE, 4.8 milliseconds; flip angle, $8^{\circ}$; section thickness, $1.0 \mathrm{~mm}$; matrix, $256 \times 256 \times 195$; FOV, $220 \times 220 \mathrm{~mm}$; voxel size, $0.94 \times 0.94 \mathrm{~mm}$ ). T2*-weighted functional images were acquired by using a gradient EPI sequence (30 oblique coronal sections; TE, 35 milliseconds; flip angle, $90^{\circ}$; section thickness, $5 \mathrm{~mm}$, with a 1 -mm gap; matrix, $80 \times 80$; FOV, $220 \times 220 \mathrm{~mm}$; voxel size, $2.75 \times 2.75 \mathrm{~mm}$ ). The 19 th anteriormost section was positioned to intersect the inferior colliculi and the cochlear nuclei in the brain stem. Each subject underwent four 6-minute resting-state runs: 2 continuous (TR, 2 seconds; 180 volumes per run; acquisition time, 1.88 seconds; no silent gap between acquisitions) and 2 sparse (TR, 12 seconds; 30 volumes per run; acquisition time, 1.88 seconds; functional acquisitions separated by approximately 10 -second silent periods free of scanner noise). The subjects were instructed to rest quietly with their eyes closed. The subjects wore a protective headset that helped to diminish SBN from the original level of $115 \mathrm{~dB}$ sound pressure level to $95 \mathrm{~dB}$ sound pressure level. The scanner coolant pump was turned off during imaging to further reduce the ambient noise level.

\section{Data Preprocessing}

Preprocessing was performed by using the SPM12 software package (http://www.fil.ion.ucl.ac.uk/spm/software/spm12) in the Matlab 7.8 programming environment (MathWorks, Natick, Massachusetts). In each run, the functional images were corrected for head motion, coregistered, normalized to the standard Montreal Neurological Institute T1 template, and spatially smoothed with an 8-mm isotropic Gaussian kernel. Section timing correction was not performed because of the discontinuous nature of the STA data.

\section{Independent Component Analysis}

To eliminate the effect of the difference in sample sizes between the STA dataset (60 volumes, labeled STA60) and CA dataset (360 volumes, labeled CA360), a third matched continuous dataset (CA60) was created by selecting 60 volumes of the CA360 set, which corresponded in time to the STA60 dataset volumes (Fig 1). Three pairs of datasets were compared. The STA60 and CA60 datasets were compared to assess any SBN effect, because they had equal numbers of volumes. A comparison of the CA60 and CA360 datasets was performed to reveal the effect of sample size, because they were equal in terms of continuous SBN effect and differed only in sample size. Finally, the STA60 and CA360 datasets were compared directly.

Spatial independent component analysis (ICA) was performed by using the Group ICA of fMRI Toolbox software (GIFT, ver. 2.0a; http://mialab.mrn.org/software/gift/). ${ }^{6}$ The ICA order of 33, approximately half of the full temporal dimension of the STA60 and CA60 data, was chosen so as not to overfit the model. $^{7,8}$ Group ICA was assessed for concatenated STA60CA60, CA60-CA360, and STA60-CA360 datasets. Independent components were extracted by using the Infomax algorithm. Twenty iterations of ICA were performed by using the ICASSO 
algorithm to verify the stability of the components. ${ }^{9}$ All individual components were scaled to represent percentage signal change. Group statistical maps of each component were generated by performing a voxelwise 1-sample $t$ test on these individual independent component maps and then thresholded at false discovery rate corrected at $\mathrm{q}<0.05$.

The resulting group components for each dataset were subsequently visually examined to determine their neural or artifactual nature. Components were considered to be gray matter components (RSN) if they were found in clusters that matched well with particular gray matter structures rather than being diffusely scattered across large regions or found in the periphery.

\section{Statistical Comparison of RSN Spatial Maps and Their Characteristics}

To compare the results of individual analyses, a paired $t$ test was performed on individual RSN maps of STA60-CA60, CA60CA360, and STA60-CA360 analyses. T-contrasts of STA60 > CA60 and CA60 $>$ STA60 were performed to explore the effect of SBN; t-contrasts of CA360 > CA60 and CA60 > CA360 were performed to explore the effect of volume number. T-contrasts of STA60 > CA360 and CA360-STA60 were performed to see how the 2 acquisition methods compared directly. Contrast maps were thresholded at the false discovery rate corrected $\mathrm{q}<0.05$.

Statistical analyses were performed by using SPSS software (ver. 19.0; IBM, Armonk, New York). The number of voxels, average percentage signal change, and maximum percentage signal change were calculated for each individual RSN of each pair-wise ICA and compared between STA60 and CA60, CA60 and CA360, and STA60 and CA360 by using the Wilcoxon signed rank test with Bonferroni correction for multiple comparisons.

\section{Functional Connectivity Analysis}

Intranetwork RSN functional connectivity strength, another measure of compositional robustness, was compared between the 3 dataset pairs. Connectivity was assessed between ROIs placed in major subregions of each RSN from each dataset. Each RSN was divided into spatially nonoverlapping subareas (eg, left/right, frontal/parietal, left (right)/medial), which resulted in several pairs of coupled subregions. The peak voxel of each subarea was used to create ROIs. ROIs were created as spheres of 3-mm radius. Center voxels for ROIs for each dataset pair comparison were chosen as peak voxels of group ICA maps performed on the corresponding concatenated datasets.

Connectivity analysis was performed by using the CONN functional connectivity toolbox, Version 15.0 ${ }^{10}$; http://www. nitrc.org/projects/conn) with the CompCor method for estimating and removing physiologic and other sources of noise. ${ }^{11}$ The individual motion parameters and a linear term for detrending were included as covariates. The residual blood-oxygen level dependent signal was bandpass-filtered over a low-frequency window of interest $(0.01-0.1 \mathrm{~Hz})$. For each dataset and for each ROI, time courses were extracted and averaged over all voxels in the ROI. Pearson correlation coefficients were calculated between the time courses of each pair of ROIs for every subject. Correlation coefficients and correlation maps were converted to Fisher $\mathrm{z}$ scores and compared pair-wise between STA60 and CA60, CA60 and CA360, and STA60 and CA360 by using a Wilcoxon signed rank test with the Bonferroni correction.

\section{Frequency Domain Analysis}

To assess the fundamental fluctuation frequency of each RSN from the CA360 dataset and to determine whether it fell within the frequency range covered by STA with its lower sampling rate, a fast Fourier transform was performed on each RSN time course produced by ICA to obtain its frequency power spectrum. Before performing the Fourier transform, a Butterworth high-pass filter was applied to all time courses, with a lower cutoff frequency of $0.01 \mathrm{~Hz}$.

\section{RESULTS}

\section{Independent Component Analysis}

All independent components of all 3 datasets had very high stability indices (mean STA60-CA60, $0.948 \pm 0.055$; CA60-CA360, $0.972 \pm 0.01$; STA60-CA360, $0.975 \pm 0.072$ ). Fourteen RSNs were obtained consistently from the 3 concatenated analyses of paired datasets: auditory, 2 default mode networks (DMNs), salience, dorsal and ventral attentional, 2 frontoparietal, 2 sensorimotor, 3 visual, and cerebellar networks. An additional anterolateral sensorimotor network was produced by STA60-CA60 and STA60-CA360 analyses, and a medial visual network was, in addition, found in CA60-CA360 analysis, which made a total of 15 discovered RSNs in all 3 dataset pairs (Fig 2; On-line Table 1).

\section{Statistical Comparison of RSN Spatial Maps and Characteristics}

A comparison of the STA60 and CA60 individual spatial maps revealed a larger auditory network in STA60. A comparison of CA60 and CA360 revealed greater salience, right frontoparietal, and 3 visual networks in the CA360 dataset. A comparison of STA60 and CA360 revealed greater posterior and anterior DMN and lateral visual networks in CA360 (On-line Fig 1).

The 3 investigated RSN characteristics (number of voxels, average and maximum percentage signal changes), a comparison of STA60 to CA60 revealed a greater voxel number for the posterior DMN in CA60. In CA60-CA360 comparison, the CA360 dataset consistently displayed significantly greater values than CA60 for several RSNs, including salience, frontoparietal, sensorimotor, and visual networks. A comparison of STA60 with CA360 showed a greater voxel number for the anterior and posterior DMN in CA360 (On-line Table 2).

\section{Functional Connectivity Analysis}

Stronger connectivity of the medial sensorimotor network was observed in STA60 compared with CA60; CA360 showed a more robust posterior DMN compared with CA60 and compared with STA60 (Table).

\section{Frequency Domain Analysis}

All RSNs obtained from CA360 exhibited dominant frequencies lower than the Nyquist frequency of the STA's sampling rate ( 0.0417 $\mathrm{Hz}$ ) (On-line Fig 2). However, some components had frequencies above the Nyquist frequency, with power comparable with that of the dominant frequency (such as salience or medial sensorimotor). 


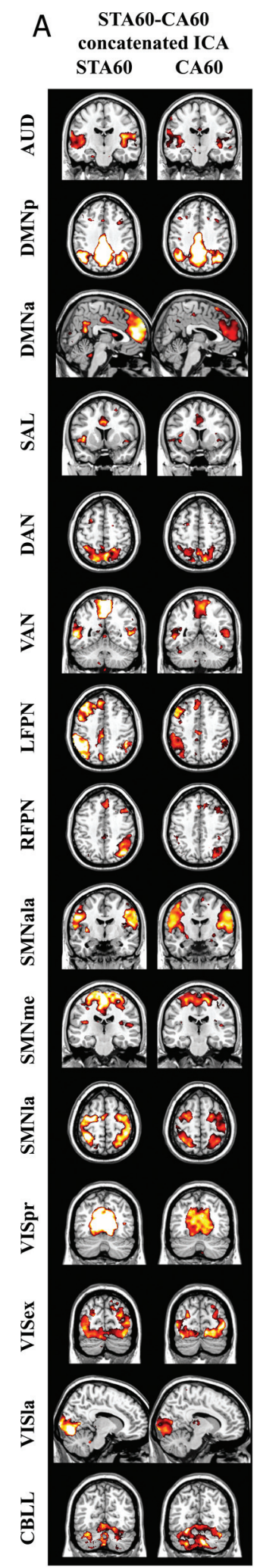

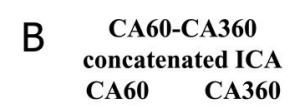

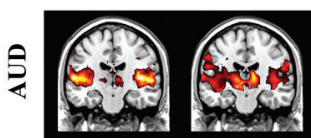

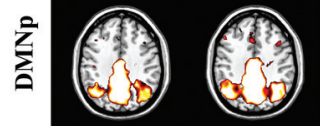

i mpos

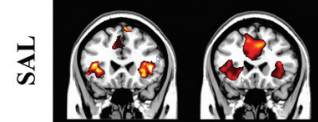

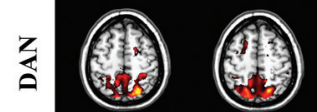

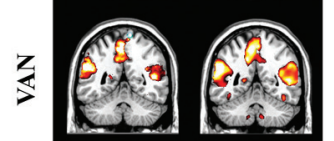

立

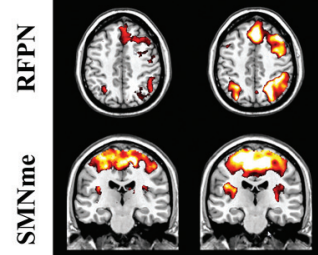

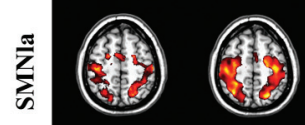

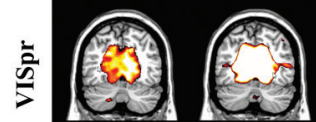
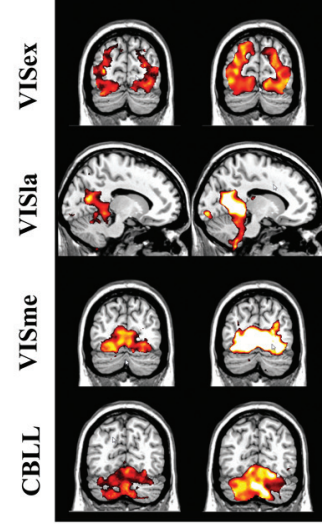

$3.5 \square 7.0$
C $\begin{array}{r}\text { STA60-CA360 } \\ \text { concatenated ICA }\end{array}$ STA60 CA360

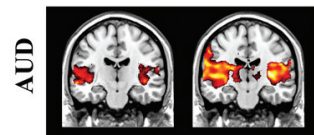

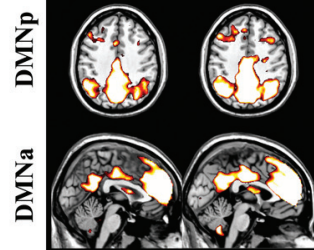

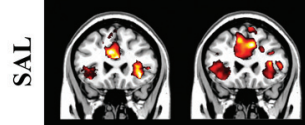

a vas

$\sum$ Fin

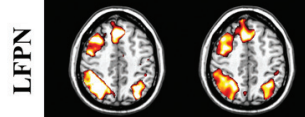

(3)

?

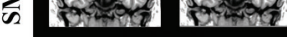

i (5x) (5)

is masio manis

$\sum_{\infty}^{5}$ (5)

$=$ axis

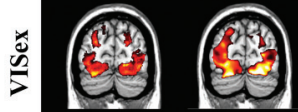

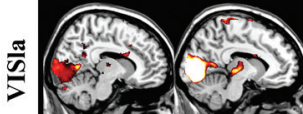

- 1 (1)

FIG 2. ICA results of each dataset pair. A, STA60-CA60 concatenated datasets. B, CA60-CA360 concatenated datasets. C, STA60-CA360 concatenated datsets. A total of 15 networks were identified in each of the analyses; 14 common networks: auditory (AUD), default mode posterior/anterior (DMNp/a), salience (SAL), dorsal/ventral attentional (DAN/VAN), right/left frontoparietal (RFPN/LFPN), sensorimotor medial/lateral (SSMme/la), visual primary/extrastriate/lateral (VISpr/ex/la), and cerebellar (CBLL) networks. In addition, the anterolateral sensorimotor network (SSMala) was identified in STA60-CA60 and STA60-CA360 analyses, and the medial visual network (VISme) was identified in CA60-CA360 analysis. Spatial maps are presented on the most representative section according to a neurologic convention thresholded at false discovery rate corrected $\mathrm{q}<0.05$. 


\begin{tabular}{|c|c|c|c|c|c|c|c|}
\hline \multirow[b]{2}{*}{ RSN } & \multirow[b]{2}{*}{ Notation } & \multicolumn{2}{|c|}{ STA60-CA60 } & \multicolumn{2}{|c|}{ CA60-CA360 } & \multicolumn{2}{|c|}{ STA60-CA360 } \\
\hline & & STA60 & CA60 & CA60 & CA360 & STA60 & CA360 \\
\hline Auditory & AUD_LR & 0.17 & 0.16 & 0.12 & 0.25 & 0.21 & 0.14 \\
\hline \multirow[t]{4}{*}{ Default mode } & DMNp_ML & -0.04 & 0.07 & 0.18 & 0.25 & 0.08 & 0.11 \\
\hline & DMNp_MR & -0.02 & 0.04 & 0.14 & 0.12 & -0.03 & 0.04 \\
\hline & DMNp_LR & 0.20 & 0.29 & 0.19 & 0.26 & 0.15 & $0.33^{\mathrm{b}}$ \\
\hline & DMNa_FP & 0.19 & 0.19 & 0.18 & 0.26 & 0.23 & 0.25 \\
\hline \multirow[t]{3}{*}{ Salience } & SAL_ML & 0.13 & 0.23 & 0.19 & 0.15 & 0.26 & 0.26 \\
\hline & SAL_MR & 0.21 & 0.23 & 0.03 & 0.08 & 0.21 & 0.27 \\
\hline & SAL_LR & 0.27 & 0.26 & -0.02 & -0.02 & 0.20 & 0.25 \\
\hline \multirow[t]{4}{*}{ Dorsal/ventral attention } & DAN_LR & 0.19 & 0.29 & 0.20 & 0.29 & 0.36 & 0.36 \\
\hline & VAN_ML & 0.27 & 0.33 & 0.19 & 0.24 & 0.06 & 0.16 \\
\hline & VAN_MR & 0.07 & 0.12 & 0.20 & 0.25 & 0.22 & 0.31 \\
\hline & VAN_LR & 0.01 & 0.11 & 0.09 & 0.16 & 0.29 & 0.37 \\
\hline \multirow[t]{2}{*}{ Frontoparietal } & RFPN_FP & 0.25 & 0.11 & 0.28 & 0.31 & 0.09 & 0.22 \\
\hline & LFPN_FP & -0.06 & -0.07 & 0.04 & 0.00 & 0.07 & 0.17 \\
\hline \multirow[t]{4}{*}{ Sensorimotor } & SMNala_LR & 0.31 & 0.13 & - & - & 0.37 & 0.27 \\
\hline & SMNme_LR & 0.25 & 0.11 & 0.27 & 0.28 & 0.21 & 0.23 \\
\hline & SMNla_LR & 0.02 & -0.01 & 0.03 & 0.13 & 0.28 & 0.31 \\
\hline & VISpr_LER & 0.37 & 0.41 & 0.03 & 0.13 & 0.68 & 0.74 \\
\hline \multirow[t]{3}{*}{ Visual } & VISex_LR & 0.39 & 0.40 & 0.02 & 0.06 & 0.34 & 0.28 \\
\hline & VISla_LR & 0.27 & 0.34 & 0.08 & 0.15 & 0.40 & 0.49 \\
\hline & VISmé_LR & - & - & 0.39 & 0.44 & - & - \\
\hline Cerebellum & CBLL_L̄R & 0.01 & -0.02 & 0.05 & 0.06 & 0.13 & 0.04 \\
\hline
\end{tabular}

Note:- L indicates left; R, right; M, medial; F, frontal; P, posterior; AUD, auditory; DMNp/a, default mode posterior/anterior; SAL, salience; DAN/VAN, dorsal/ventral attentional; RFPN/LFPN, right/left frontoparietal; SSMala/me/la, sensorimotor anteromedial/medial/lateral; VISpr/ex/la/me, visual primary/extrastriate/lateral/medial; CBLL, cerebellar networks.

${ }^{a}$ Correlation coefficients between subareas of each RSN were compared for presenting SBN effect (STA60-CA60 comparison), sample size effect (CA60-CA360 comparison), and the direct comparison of the 2 acquisition methods (STA60-CA360 comparison).

${ }^{\mathrm{b}}$ Significantly greater than the comparison value of the other dataset $(P<.05$, Bonferroni corrected, Wilcoxon signed rank test).

\section{DISCUSSION}

The issue of SBN effects on the resting state of the brain remains poorly explored. To date, 2 reported studies evaluated the SBN effect on the resting state. Rondioni et $\mathrm{al}^{12}$ used a "silent" EPI sequence, which allowed reduction of SBN by only approximately $12 \mathrm{~dB}$. Although quieter than the original EPI SBN level, this does not seem to provide an adequately "silent" environment suitable for auditory studies. In our study, STA provided approximately 10-second completely silent periods between consecutive image acquisitions, whereas SBN was generated continuously during CA sessions. Another study used STA in comparison with the CA technique for rest and auditory task conditions. ${ }^{13}$ Although the researchers used ICA as in this study, there were significant differences: first, we observed and included into the analysis substantially more RSNs for comparison; second, we eliminated the influence of a greater sample size of CA by creating the volume-matched CA60 dataset.

ICA successfully extracted consistent canonical RSNs identified by previous resting-state and task-based studies for each pair of datasets. ${ }^{14-17}$ Except for an anterolateral sensorimotor network in STA60-CA60 and STA60-CA360 analyses, and the medial visual network in CA60-CA360 analysis, all identified RSNs matched across the 3 dataset pairs. RSNs identified from all dataset pairs included most of the RSNs provided as templates with the GIFT software ${ }^{18}$ and RSNs identified by a multisubject high-order ICA study. ${ }^{17}$ All components were highly stable.

A comparison of the STA60 with the CA60 dataset revealed the larger number of voxels for the posterior DMN in CA60 (On-line Table 2). No other differences were found, either in spatial RSN characteristics or in the intranetwork functional connectivity (Table). Therefore, SBN did not seem to affect RSNs significantly.
The CA360 dataset, however, was significantly larger than CA60 in terms of the voxel number and average and maximum percentage signal changes for a number of networks. These results were in line with the comparison of RSN spatial maps, in which the same RSNs had a significantly greater spatial extent in CA360 than those in CA60 (On-line Fig 1). This could indicate that the larger sample size of CA360 had a substantial effect on the spatial distribution of the ICA components. However, the sample size did not appear to have any impact on functional connectivity because no differences between the 2 datasets were found.

Direct comparison of the STA60 with the CA360 dataset revealed a larger number of voxels in the anterior and posterior DMN in CA360 (which agreed with the results of the spatial maps comparison) (On-line Table 2; On-line Fig 1). CA360 also demonstrated stronger functional connectivity for the posterior DMN (Table). Because the effects of SBN and sample size were not separated here, it is unclear how much either was responsible for this difference. Although a larger sample size appeared to have a stronger influence on ICA results after the results of CA60-CA360 comparison, the posterior DMN demonstrated a higher voxel number in CA60 compared with STA60, which means that a larger DMN in CA360 may also be due to the SBN effect. The brain switches into the default mode of ongoing introspective activity when it is not engaged in any immediate attention-demanding task or stimulation. ${ }^{19,20}$ In fMRI resting-state studies, there is no active task or stimulus to voluntarily direct attention, which can result in attention being involuntarily drawn to SBN.

Continuous SBN in CA, if recognized as a constant but meaningless and harmless sensory stimulus, would lead to attention system fatigue and send top-down controlling signals to the pe- 
ripheral auditory system to discontinue responding. ${ }^{21}$ Decreased attention and adaptation (fatigue) to the continuous stimulus (SBN) in CA may result in increased activity in the default mode network. In contrast, the far sparser occurrence of SBN in STA, similar to auditory oddball or novelty stimulation, might evoke an attentional response, which, subsequently, may keep subjects more alert and result in disturbed default mode activity. Note that this might not be the case with task-oriented studies, in which allocation of attentional resources is largely governed by the task at hand, and SBN may not impact attentional and default mode networks in the same manner as in the resting state. Furthermore, although the described mechanism related to regulation of attention by SBN is one possible explanation, other scenarios may exist and should not be ruled out.

Frequency analysis showed that the dominant frequency of all RSNs (which is known to be $<0.1 \mathrm{~Hz}^{22,23}$ ) was lower than the Nyquist frequency of the STA's sampling rate $(0.0417 \mathrm{~Hz})$, which indicated that the low sampling rate of STA was fast enough to capture low-frequency fluctuations of resting brain networks. However, some components of CA360 had frequencies beyond the Nyquist frequency, which had a comparably strong power. It means that some essential information about temporal dynamics could still be lost in STA, which is a method far inferior to CA for studying temporal behavior of the brain because of its discontinuous nature.

Altogether, SBN did not seem to have any effect on RSN spatial maps and connectivity, except possibly disturbing DMN activity. Thus, CA should be a preferred method of acquisition in general resting-state studies due to its superior temporal resolution, increased statistical power, and less subject-to-subject variability. STA, however, did not appear to have any benefits compared with CA. Its disadvantages, such as increased variability, temporal discontinuity, small sample size, and decreased power, make it a far inferior method to CA. STA may be applicable only in very rare and particular cases, such as when continuous SBN may mask tinnitus or is unbearable by patients with hyperacusis or other auditory disorders.

Regarding the limitations of the current study, it should be noted that SBN may not be the only difference factor between STA60 and CA60 datasets. The 2 datasets may differ in signal-tonoise ratio because the longer interacquisition intervals in STA allow for better T1 recovery and thus greater signal intensity. ${ }^{5}$

\section{CONCLUSIONS}

STA was able to extract the same networks as CA, but it did not display any advantages over the continuous method. SBN did not seem to affect either spatial maps or connectivity of the RSNs. Therefore, apart from very particular cases when continuous SBN is unwanted or unbearable, CA is a recommended acquisition method for most of the resting-state studies, including those of auditory disorders, due to its eminent temporal resolution and high statistical power.

\section{REFERENCES}

1. Maudoux A, Lefebvre P, Cabay JE, et al. Auditory resting-state network connectivity in tinnitus: a functional MRI study. PLoS One 2012;7:e36222 CrossRef Medline
2. Ueyama T, Donishi T, Ukai S, et al. Brain regions responsible for tinnitus distress and loudness: a resting-state FMRI study. PLoS One 2013;8:e67778 CrossRef Medline

3. Schmidt SA, Akrofi K, Carpenter-Thompson JR, et al. Default mode, dorsal attention and auditory resting state networks exhibit differential functional connectivity in tinnitus and hearing loss. PLoS One 2013;8:e76488 CrossRef Medline

4. Burton H, Wineland A, Bhattacharya M, et al. Altered networks in bothersome tinnitus: a functional connectivity study. BMC Neurosci 2012;13:3 CrossRef Medline

5. Hall DA, Haggard MP, Akeroyd MA, et al. "Sparse" temporal sampling in auditory fMRI. Hum Brain Mapp 1999;7:213-23 Medline

6. Calhoun VD. Group ICA of fMRI toolbox (GIFT). 2004. http:// mialab.mrn.org/software/. Accessed October 15, 2015.

7. Abou Elseoud A, Littow H, Remes J, et al. Group-ICA model order highlights patterns of functional brain connectivity. Front Syst Neurosci 2011;5:37 CrossRef Medline

8. Kiviniemi V, Starck T, Remes J, et al. Functional segmentation of the brain cortex using high model order group PICA. Hum Brain Mapp 2009;30:3865-86 CrossRef Medline

9. Himberg J, Hyvarinen A. Icasso: software for investigating the reliability of ICA estimates by clustering and visualization. In: Proceedings of the IEEE 13th Workshop on Neural Network for Signal Processing, Toulouse, France. May 17-19, 2003: 259-68

10. Whitfield-Gabrieli S, Nieto-Castanon A. Conn: a functional connectivity toolbox for correlated and anticorrelated brain networks. Brain Connect 2012;2:125-41 CrossRef Medline

11. Behzadi Y, Restom K, Liau J, et al. A component based noise correction method (CompCor) for BOLD and perfusion based fMRI. $\mathrm{Neu}$ roimage 2007;37:90-101 CrossRef Medline

12. Rondinoni C, Amaro E Jr, Cendes F, et al. Effect of scanner acoustic background noise on strict resting-state fMRI. Braz J Med Biol Res 2013;46:359-67 CrossRef Medline

13. Langers DR, van Dijk P. Robustness of intrinsic connectivity networks in the human brain to the presence of acoustic scanner noise. Neuroimage 2011;55:1617-32 CrossRef Medline

14. Beckmann CF, DeLuca M, Devlin JT, et al. Investigations into resting-state connectivity using independent component analysis. $\mathrm{Phi}$ los Trans R Soc Lond B Biol Sci 2005;360:1001-13 CrossRef Medline

15. Damoiseaux JS, Rombouts SA, Barkhof F, et al. Consistent restingstate networks across healthy subjects. Proc Natl Acad Sci U S A 2006;103:13848-53 CrossRef Medline

16. De Luca M, Beckmann CF, De Stefano N, et al. fMRI resting state networks define distinct modes of long-distance interactions in the human brain. Neuroimage 2006;29:1359-67 CrossRef Medline

17. Allen EA, Erhardt EB, Damaraju E, et al. A baseline for the multivariate comparison of resting-state networks. Front Syst Neurosci 2011; 5:2 CrossRef Medline

18. Shirer WR, Ryali S, Rykhlevskaia E, et al. Decoding subject-driven cognitive states with whole-brain connectivity patterns. Cereb Cortex 2012;22:158-65 CrossRef Medline

19. Raichle ME, Snyder AZ. A default mode of brain function: a brief history of an evolving idea. Neuroimage 2007;37:1083-90; discussion 1097-99 CrossRef Medline

20. Buckner RL, Andrews-Hanna JR, Schacter DL. The brain's default network: anatomy, function, and relevance to disease. Ann N Y Acad Sci 2008;1124:1-38 CrossRef Medline

21. Guinan JJ Jr. Cochlear efferent innervation and function. Curr Opin Otolaryngol Head Neck Surg 2010;18:447-53 CrossRef Medline

22. Greicius MD, Krasnow B, Reiss AL, et al. Functional connectivity in the resting brain: a network analysis of the default mode hypothesis. Proc Natl Acad Sci U S A 2003;100:253-58 CrossRef Medline

23. Fransson P. Spontaneous low-frequency BOLD signal fluctuations: an fMRI investigation of the resting-state default mode of brain function hypothesis. Hum Brain Mapp 2005;26:15-29 CrossRef Medline 